\title{
Segmentation-based Path Switching Mechanism for Reduced Data Losses in OBS Networks
}

\author{
Anna V. Manolova ${ }^{1}$, Jakob Buron ${ }^{1}$, Sarah Ruepp ${ }^{1}$, Lars Dittmann ${ }^{1}$, \\ and Lars Ellegard ${ }^{2}$
}

${ }^{1}$ COM•DTU, Oersteds Plads, Building 345V, Technical University of Denmark, 2800 Kgs. Lyngby, Denmark, tel:+45 45256609

2 Tellabs Denmark A/S, Lautrupvang 3C, 2750 Ballerup, Denmark

$\{$ avm, jbu, sr, ld\}@com.dtu.dk

lars.ellegard@tellabs.com

\begin{abstract}
The Optical Burst Switching (OBS) technology emerged as an alternative switching paradigm for the optical transport layer. Its biggest disadvantage (high data losses) has been the focus of numerous research papers. This paper proposes a data loss reduction technique, which relies on the combination of global network coordination between network nodes and local contention resolution. Via full-scale network simulation it is demonstrated that the proposed scheme has improved performance in terms of data losses and resource utilization, compared to its constituent mechanisms alone. Furthermore, the effectiveness of the mechanism has proven to be less sensitive to load variations in the medium and high load ranges. Additionally, its complexity and deployment cost are low, due to the absence of immature optical components in the network.
\end{abstract}

Keywords: OBS, segmentation, load balancing, contention resolution

\section{Introduction}

Optical Burst Switching (OBS) is an emerging technology introduced approximately six years ago. In one of the first publications [1], the technology is described as a promising solution for the direct mapping of the bursty IP traffic over the optical transmission media (IP over WDM). There are numerous advantages of OBS over the traditional packet switching (OPS) and channel switching (OCS) paradigms. OBS uses medium granularity of the switching entities (bursts) and resides between OCS and OPC. Its specific way of operation results in bypassing the existing bottleneck in the electronic routers and poses more moderate requirements for optical buffering. OBS is highly dynamic, and achieves a great degree of statistical multiplexing. Additionally, the technology provides complete transparency of the switching node regarding the code, the format and the speed of the client traffic and the used upper-layer protocols by providing all-optical transport of the client data.

The basic idea of the OBS network is fairly simple [2]. At the edge of the OBS domain the client data is assembled into bursts depending on its destination. 
Each burst sent in the network is preceded by a special Burst Header Packet (BHP), which carries all needed routing and signaling information and uses a dedicated Control Channel. The burst is released in the network with or without waiting for an acknowledgment from the egress node. In the former case a oneway resource reservation is used, i.e. this OBS network is a "best-effort" type of network. The latter uses Tell-and-Wait (TAW) resource reservation [2], making the OBS network effectively connection-oriented. The majority of research work, as well as this paper, focuses on connectionless OBS networks. Despite its obvious advantages, the OBS technology is still unapplicable in real optical networks because it suffers from one major disadvantage: increased data losses. This paper discusses this issue and proposes an effective loss reduction technique called Segmentation-based Path Switching (SPS).

The rest of this paper is organized as follows. Section 2 offers an overview of the stated problem and summarizes the existing solutions found in the literature. Section 3 analyzes the performance of two contention resolution techniques and introduces the SPS mechanism. Section 4 provides numerical results obtained from simulations and their analysis. Section 5 concludes the paper.

\section{Data Losses in OBS Networks}

Contention refers to the situation, when two or more bursts request the same resource at the same time and is the main source of data losses in an OBS network. In connectionless OBS networks the contention issue is severe due to the the lack of optical memory and the applied one-way resource reservation technique, which does not guarantee burst delivery. In fact, the contention resolution problem involves many different aspects such as routing, signaling, channel scheduling, burst assembly and available hardware components.

There are basically two classes of contention resolution techniques: reactive and proactive. The reactive techniques aim at minimizing the losses after a contention has occurred. The main strategy is deflection, which can occur in time (buffering), space (deflection routing) or/and frequency (wavelength conversion) domains. Another strategy is the so called soft contention resolution policy. Such policies are Segmentation [3], look-ahead contention resolution and shortest/latest drop policies [4]. The proactive mechanisms try to avoid congestion in the network by applying smart traffic routing and balancing [5], admission control [6], and optimal burst assembly (e.g. composite burst assembly [3]).

In both proactive and reactive mechanisms the trade-off between complexity and efficiency is obvious. The contention resolution strategies are considered to be highly dependent on the traffic load [7], in particular the deflection routing strategy, which tends to decrease severely in performance when the network load increases. The proactive solutions involve feedback information distribution and/or sophisticated algorithms for smart routing and traffic shaping, which increase the complexity in the network.

Most of the research work done in this area focuses separately on prevention and resolution schemes. In this paper a combination of two mechanisms is of- 
fered. The operation of the mechanism is described in the next section and its performance is tested via the OPNET Modeler software [8].

\section{Loss Reduction Techniques in OBS Networks}

Even though the contention resolution mechanisms suffer from decreased performance at high loads they are an integral part of the overall effort to reduce the packet loss ratio in an OBS network. In order to overcome this drawback we propose to combine a reactive contention resolution scheme with a proactive scheme, which will address this problem. This scheme is called Segmentationbased Path Switching (SPS) and combines the Segmentation contention resolution ( $\mathrm{S}$ ) technique, proposed in [3], and the Congestion-Based Static-Route Calculation contention prevention (CP) scheme, proposed in [5]. S and CP are referred to as the constituent mechanisms of SPS. They are chosen because of their simplicity and because they do not require wavelength convertors or fiber delay lines. This makes the SPS mechanism low-cost and relatively simple.

\subsection{Evaluation of the Constituent Loss Reduction Techniques}

The Segmentation technique was first proposed in [9]. Its basic operation is as follows: when two bursts contend for resources, depending on the applied policy, only part of one of the bursts is dropped instead of dropping a whole burst. The authors of [9] offer to drop the head of the later-arriving burst to be dropped, whereas the authors of [3] suggest to drop the tail of the earlier-arriving burst. The technique has been further investigated in several research papers and has proven to be effective, but all results confirm the fact that at high loads the mechanism's performance degrades [3], [10], [11].

The CP mechanism, proposed in [5], needs a congestion situation (i.e. higher loads) in the network in order to be efficient. Each ingress node statically calculates two link disjoint paths towards each destination. Each core node periodically floods link-status advertisements, indicating whether the load on its outgoing links has passed a certain threshold. When there is a burst ready to be sent, the ingress node calculates the congestion level of the overall path and chooses the least congested path to the destination. There are two important parameters influencing the performance of this scheme: the threshold value, which indicates when a link is considered to be overloaded, and the observation period, over which the load status of a link is calculated. It must be noted that the performance of this CP technique is dependent on the network topology. In general, schemes which use adaptive routing techniques are dependent on the connectivity in the network and the overall topology. Nevertheless, the overall performance in terms of data losses is improved due to the load balancing achieved in the network. The biggest disadvantage of this scheme is that it does not support different classes of service.

Both mechanisms involve additional signaling in the network. In case Segmentation is applied, the downstream nodes are informed about the change in 
the burst length via update packets. The more segmentations there are in the network, the higher the signaling overhead will become. The CP mechanism is a feedback-based mechanism, which means that feedback information is constantly exchanged. When the network load increases it is obvious that feedback information will be exchanged more often.

\subsection{Combined Segmentation-based Path Switching Technique}

Our proposed loss reduction scheme combines the best of both described mechanisms: the contention prevention is responsible for better load balancing in the network, while the contention resolution reactively reduces the packet losses. Unlike the scheme proposed in [12], which also consists of global and local loss reduction strategies, the SPS scheme does not require fiber delay lines, wavelength convertors or complicated channel scheduling algorithms. This makes our strategy applicable in the near future and reduces the capital expenditure of deployment.

The complexity of SPS is distributed among two main modules: a local contention resolution module (LCRM) and a global contention prevention module (GCPM). LCRM executes the Segmentation and only has knowledge of the local node condition, whereas GCPM has a global overview of the network status which is stored in every node and is updated periodically. These two modules are completely independent, which increases the reliability of the mechanism. They have a different scope of work and a failure in one of them will not disturb the operation of the other. Each module is also related to a different plane of the OBS network. The GCPM operates on the control plane of the network by affecting the routing and the offset-time management, whereas the focus of the LCRM is on the data plane. This clear separation of both modules is compliant with the separation of the data and control planes of the OBS network and makes the mechanism fit properly in the overall OBS architecture (see Fig. 1).

The independence of both constituent mechanisms allows the SPS to fully exploit one significant advantage - support for different class differentiation techniques. Class differentiation can be done by using the built-in class support of the Segmentation technique [3]. It offers class differentiation based on prioritization of the traffic and by applying different segmentation policies. Another option is introducing differentiated class handling based on the $\mathrm{CP}$ scheme. For example only high priority class traffic can be switched to the secondary path in case of a congested primary one, or different link and node disjoint paths can be calculated separately for delay and for loss sensitive traffic. Since nothing prevents different combinations of class differentiating schemes, SPS turns into a highly flexible class-supporting mechanism. At this stage, the proposed SPS technique accommodates only the built-in Segmentation capabilities. The suggested CP-based methods are subject to further study. 


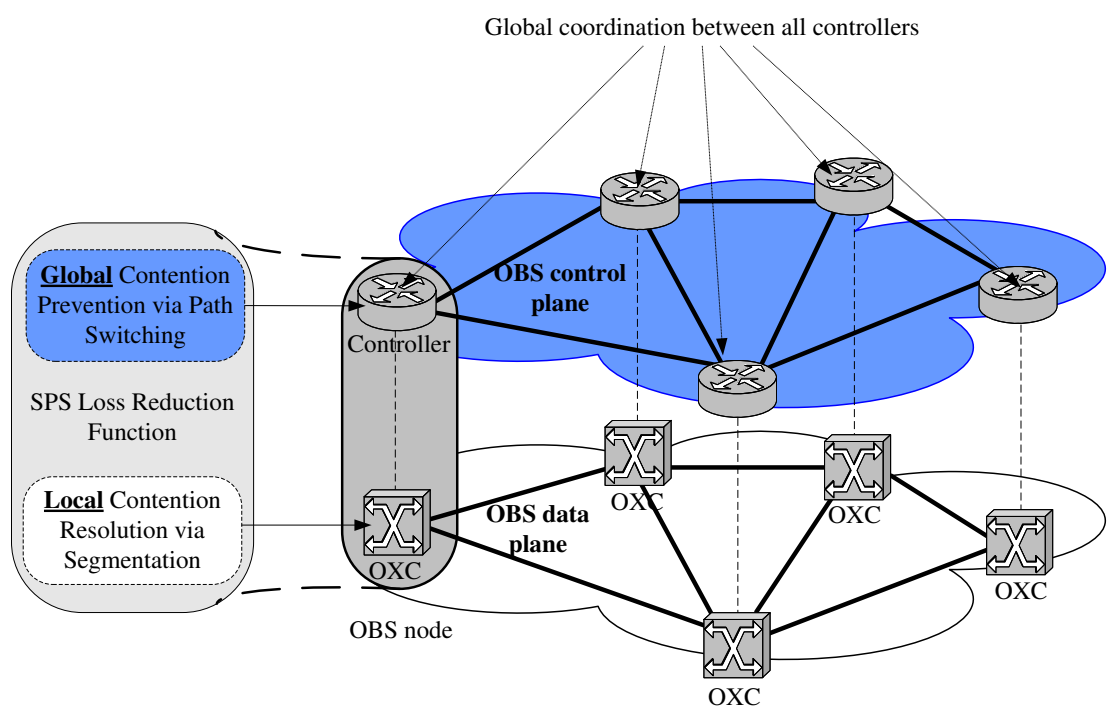

Fig. 1. Separation of the different modules in the SPS mechanism.

\section{Numerical Results}

In this section, the results obtained through OPNET simulations are presented. A model of an OBS node (see Fig. 2 (a)) was applied in the NSFNET network, shown in Fig. 2 (b). Each OBS node can serve as both Edge and Core node. There are four wavelengths for data transfer and one for the signaling channel on each optical fiber. No wavelength conversion, nor fiber delay lines are employed. Based on [3], the BHP processing time is chosen to be $2.5 \mu \mathrm{s}$, the cross-connect configuration time is $10 \mu$ s and upon contention, the tail of a burst is segmented and dropped. Each data burst consists of 100 segments and has a fixed length of $100 \mu \mathrm{s}$. The burst arrival process has exponential distribution and the generated traffic is uniformly distributed among all sender-receiver pairs. Minimum Delay Shortest Path routing is used for path computation. Whenever Segmentation is applied, the minimum allowed burst length is chosen to be 10 segments. The threshold value for the $\mathrm{CP}$ part of the mechanism is 0.5 and the observation period (i.e. the period between updates for the status of a link) is $5 \mathrm{~ms}$. Each data point is obtained after 30 seconds of simulation.

The performance of the SPS mechanism and its constituent mechanisms (Segmentation and $\mathrm{CP}$ ) will be compared to a Basic Case (BC), in which no loss saving techniques are used. The main performance measures are the Packet Loss Ratio (PLR), the End-to-End Delay (E2ED), the Resource Utilization (RU) and the Improvement Factor (IF) of the mechanisms. In the following, PLR will be used instead of BLR, because of the ambiguity of BLR when some packets are segmented out of a burst during transmission. The presented results will not in- 


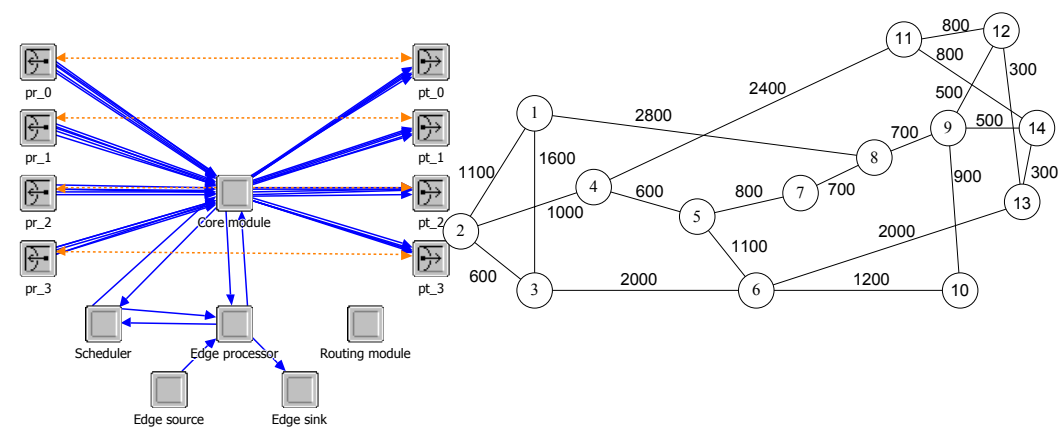

(a)

(b)

Fig. 2. (a) OBS node model and (b) NSFNET with 14 nodes and 21 bidirectional links (numbers indicate distance in $\mathrm{km}$ ).

clude class differentiation because the scope of this paper is limited to the general SPS performance compared to the performance of the constituent mechanisms. Due to the same reason, none of the earlier-specified mechanisms' parameters are be varied during simulations. It is clear that the performance of all of the mechanisms is dependent on these values, but using constant values will give a common ground for an objective and clear evaluation and comparison.

\subsection{Packet Loss Ratio}

The PLR values for all tested mechanisms and for the BC can be observed in Fig. 3. It is clearly visible that our proposed mechanism achieves the lowest PLR for medium and high loads. It is interesting to observe that the PLR characteristic of the SPS mechanism resembles the one for the CP scheme. At low loads the CP mechanism causes insignificant change in the PLR value, compared to the $\mathrm{BC}$ and for some loads its performance is even worse. This is due to the fact that the alternative link-disjoint paths are almost always significantly longer than the primary ones, which increases the probability for contention along the paths. In the middle load range, the operation of the mechanism stabilizes and the benefit of the load balancing becomes visible. This effect can also be observed for the SPS mechanism, which means that at low loads the combined SPS approach is not as good as the Segmentation one. It must be mentioned that the PLR for the SPS mechanism is strongly influenced by the values of the threshold and the observation periods of the GCPM part of the mechanism and the applied Segmentation policy for the LCRM part. 


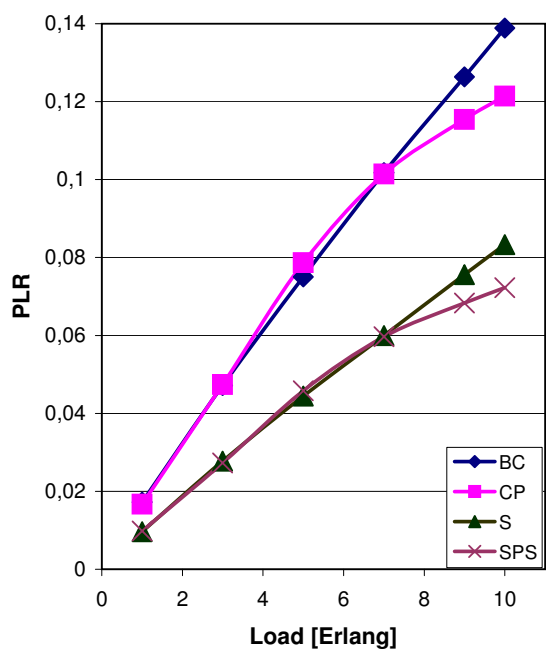

(a)

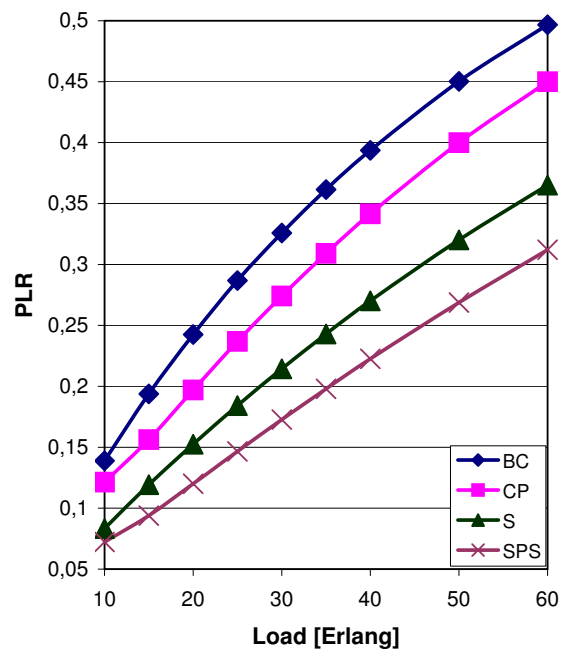

(b)

Fig. 3. PLR for low (a) and high (b) loads for all tested loss reduction mechanisms.

\subsection{End-to-End Delay and Resource Utilization}

Applying path switching will inevitably result in increased E2ED value and increased RU. A high RU value does not necessarily indicate improved network performance, since a dropped data burst close to the receiver will increase the RU. Figure 4 shows the E2ED and the RU values for all tested cases throughout the entire tested load range. The first noticeable result is the increased E2ED for all applied loss reduction mechanisms. There are two contributing components: the longer alternative paths, used in the CP and the SPS mechanisms, and the fact that bursts, which need to pass more nodes along their way to the destination, have a higher chance of surviving in the cases where Segmentation is applied. This results in the highest E2E Delay for the SPS mechanism. The higher value of the RU for the Segmentation and the SPS mechanisms indicate more saved bursts in the network, compared to the $\mathrm{CP}$ and the BC (see Fig. 3). From the figure it can be concluded that the improved PLR parameter for the SPS mechanism does not come at the expense of increased resource utilization. In fact, for the middle load range the RU parameter is slightly lower (about 1\%). This, combined with the lower PLR value, indicates more proper resource utilization.

\subsection{Improvement Factors of the Investigated Mechanisms}

The IF parameter, calculated according to (1), is a relative performance measure, and indicates a given mechanism's improvement or reduction in PLR compared 


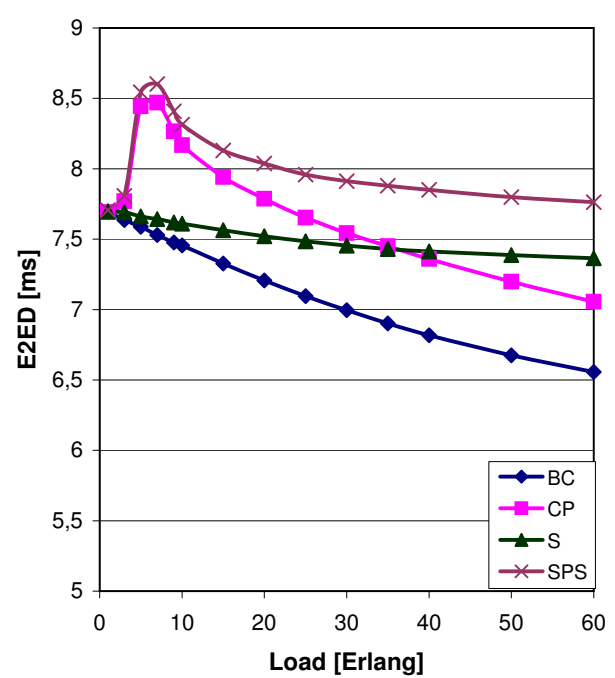

(a)

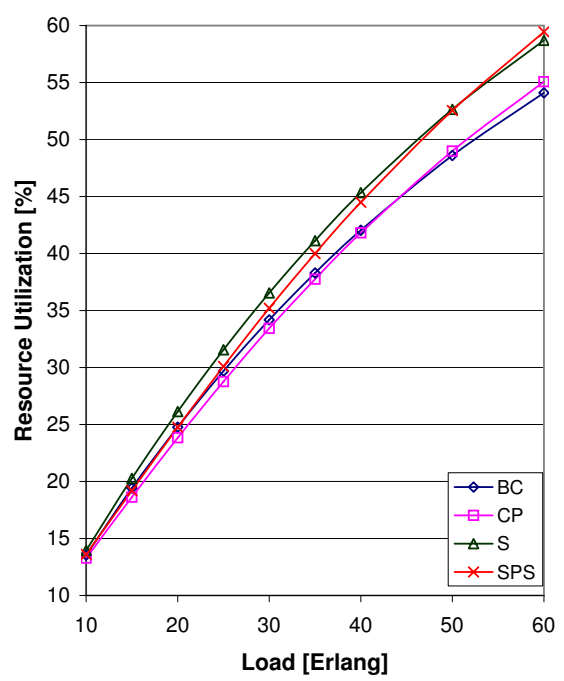

(b)

Fig. 4. End-to-End Delay (a) and Resource Utilization (b) vs. Load for all simulated mechanisms.

to the Basic Case.

$$
I F_{i}=\frac{P L R_{B C}-P L R_{i}}{P L R_{B C}} * 100[\%],
$$

$I F_{i}$ - the improvement factor of a given mechanism $i ; i \in\{$ Segmentation, CP, SPS $\}$.

The IF for all three loss saving mechanisms is presented in Fig. 5 (a). From the figure it can be seen that for medium loads the CP mechanism has increasing performance factor and for the low and very high loads the performance of the mechanism degrades. This also applies to the SPS technique. Assuming the improvement, achieved by the SPS mechanism, is the maximum gain that can be achieved, it can be seen that the Segmentation technique can attain more than $70 \%$ of this maximum gain. If the increased complexity in the network does not justify the remaining $30 \%$ gain of the improvement factor, then the Segmentation technique is the best choice. However, according to Fig. 4 (b), the SPS mechanism has more effective resource utilization and if this is the main performance measure, the SPS mechanism should be preferred. The last observation is that the performance of all mechanisms degrade at very high loads. From the results presented in Fig. 5 (a), it can be concluded that the SPS mechanism is the most appropriate for medium and high loads, and for the low load range, one should consider Segmentation only.

Since the SPS mechanism is a combination of two mechanisms, one can expect that adding up the improvement factors achieved by the component mechanisms separately, should result in a higher improvement factor than the one achieved 
by the combined scheme. This comes from the fact that some bursts can be saved with either of the two mechanisms, which reduces the improvement from adding the second mechanism. Figure 5 (b) illustrates this expectation for medium and high loads. At very high loads the IF of the SPS mechanism is actually higher than the sum of the IFs for the component schemes, meaning that at very high loads both mechanisms reinforce each other's performance. The last performance

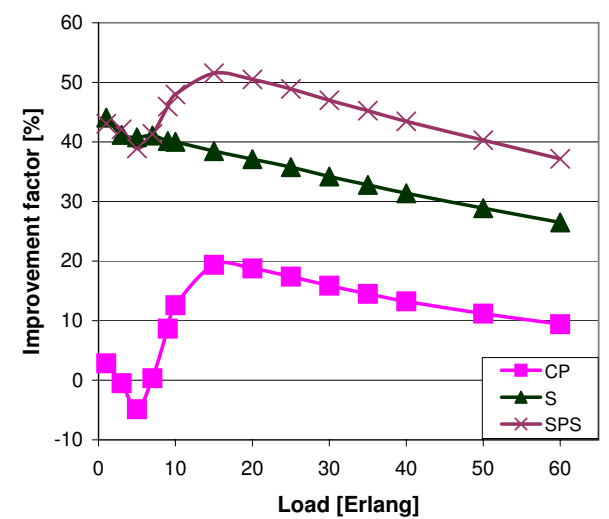

(a) Improvement Factor

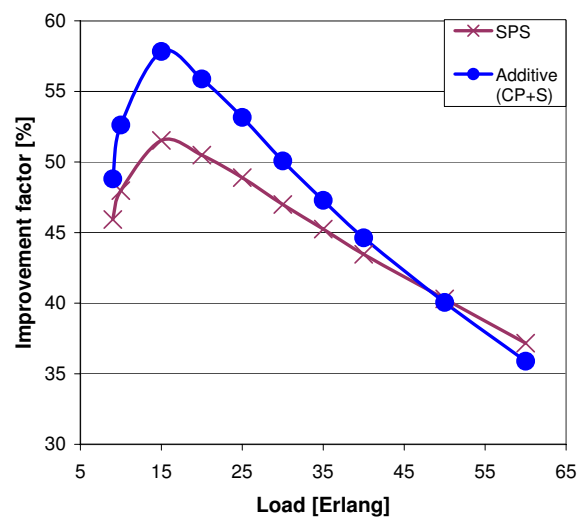

(b) SPS and additive improvement factors

Fig. 5. Improvement factors vs. Load.

evaluation technique is the comparison of the speed of degradation of the mechanisms. Applying a simple linear approximation to the PLR graphs (Fig. 3 (b)) and calculating the inclination angle of the approximation gives the speed of the PLR increase, which is an indication of the speed of the degradation of the mechanisms' performance. The obtained results for the inclination angles are:

- $33.17^{\circ}$ for the CP technique,

- $29.41^{\circ}$ for the Segmentation technique,

- $25.63^{\circ}$ for the SPS technique.

It can be seen, that the SPS mechanism has the smallest value of the inclination angle and thus the slowest speed of degradation, whereas the CP and the Segmentation mechanisms degrade faster. This is a very strong advantage of the SPS mechanism, as it indicates that the mechanism will be more stable under dynamic load conditions, compared to its component mechanisms alone.

\section{Conclusion}

In this paper a combined loss reduction strategy for OBS networks, called SPS, is proposed. The mechanism combines a local contention resolution mechanism 
(Segmentation) and a global contention prevention mechanism (CongestionBased Static-Route Calculation). Its performance is evaluated in realistic IPover-WDM full-scale network scenario. The technique overcomes the disadvantages of its constituent mechanisms and leverages their strengths at the expense of slightly increased signalling in the network. Our approach does not require wavelength conversion, fiber delay lines for buffering or complex channel scheduling algorithms and is hence more eligible for deployment in the near future. SPS shows improved PLR, better utilization of resources for medium and high loads, and offers great flexibility for differentiated traffic handling. At low loads the achieved improvement does not justify the increased complexity in the network and the Segmentation strategy alone proves to be more efficient. In addition, the mechanism proved to be less susceptible to traffic load variations for medium and high loads, which makes it suitable for dynamic traffic patterns. The conducted simulations reveal that the SPS mechanism is most appropriate to be used at the middle load ranges, where the effectiveness of the mechanism is the highest and the resource utilization is optimal.

\section{References}

1. Qiao, C., Yoo, M.: Optical Burst Switching (OBS) - A New Paradigm for an Optical Internet. J. High Speed Networks, Vol. 8, No. 1, pp. 69 - 84, 1999.

2. Jue, J.P., Vokkarane, M.V.: Optical Burst Switched Networks. Springer Science + Business Media Inc., 2005.

3. Vokkarane, M.V., Jue, J.P.: Prioritized Burst Segmentation and Composite BurstAssembly Techniques for QoS Support in Optcal Burst-Switched Networks. IEEE Journal on Selected Areas in Communications, Vol. 21, No. 7, September 2003.

4. Farahmand, F., Jue, J.P.: Look-ahead Window Contention Resolution in Optical Burst Switched Networks. IEEE Workshop on HPSR, Italy, pp. 147 - 151, 2003.

5. Thodime, G., Vokkarane, M.V., Jue, J.P.: Dynamic Congestion-Based Load Balanced Routing in Optical Burst-Switched Networks. In Proc. of Globecom 2003, pp. $2628-2632$.

6. Maach, A., Bochmann, G.V., Mouftah, H.: Congestion Control and Contention Elimination in Optical Burst Switching. Telecommunication Systems Journal, Vol. 27, No. 2, pp. 115 - 131, October 2004.

7. Gauger, C.M., Kohn, M., Schafr, J.: Comparison of Contention Resolution Strategies in OBS Network Scenarios. ICTON 2004, Wroclaw, Poland, July 2004.

8. OPNET Modeler Educational Version 11.5, http://www.opnet.com

9. Detti, A., Vramo, E., Listanti, M.: Performance Evaluation of a New Technique for IP Support in a WDM Optical network: Optical composite Burst Switching (OCBS). J. Lightwave Technol., Vol. 20, No. 2, February 2002.

10. Anpeng, H., Linzhen, X.: A Novel Segmentation and Feedback Model for Resolving Contention in Optical Burst Switching. Photonic Network Communications, $6: 1$, $61-67,2003$.

11. Zalesky, A. et al.: OBS Contention Resolution Performance. Performance Evaluation (2006), doi:10.1016/j.peva.2006.06.002

12. Wen, H., Song, H., Li, L., Wang, S.: Load Balancing Contention Resolution in OBS Networks Based on GMPLS. J. High Performance Computing and Networking, Vol. 3, No. 1, 2005. 